\title{
Fatores que interferem na adesão ao tratamento da hipertensão arterial sistêmica em pacientes atendidos no Núcleo de Atendimento ao Hipertenso (NAHI) e no Programa Saúde da Família (PSF), no município de Barbacena
}

\author{
Factors that interfere in the hypertension treatment \\ envolvement of patients in the NAHI - Núcleo de \\ Atendimento ao Hipertenso (Center of Support to the Hand in \\ the PSF - Programa Saúde da família (Family Health Program)
}

\author{
Carla Alpha Gilsogamo ${ }^{1}$ \\ Juliana Cristina de Oliveira ${ }^{1}$ \\ Julio César Amaral Teixeira ${ }^{1}$ \\ Leandro Coelho Negri Grossi ${ }^{1}$ \\ Marcos Monteiro Pinto Moreira ${ }^{1}$ \\ Leonor Oliveira Diniz ${ }^{2}$
}

\section{Resumo}

OBJETIVOS: determinar o conjunto de fatores que interferem na adesão ao tratamento de pacientes com hipertensão arterial sistêmica atendidos em um serviço especializado: NAHI (Núcleo de Atendimento ao Hipertenso) e no PSF (Programa de Saúde da Família) no município de Barbacena.

METODOS: trata-se de um estudo de coorte transversal realizado através de inquérito (questionário contendo 46 questões).

RESULTADOS: Foram coletados 154 questionários no NAHI e 246 no PSF. De acordo com a resposta ao item 27 do questionário, 101 (25,2\%) pacientes foram incluídos na categoria não aderente e 299 (74,7\%) no grupo que adere ao tratamento. A média de idade dos entrevistados que aderem é de 58,7 anos (DP: 11,7) e 58,3 anos (DP: 12,7) para o grupo dos que não aderem. A maioria dos hipertensos não faz exercício físico. No grupo aderente, 40,5\% conseguem seus medicamentos na unidade de tratamento contra 46,5\% do grupo não aderente. Cerca de $71 \%$ dos pacientes afirmam ter dificuldade na aquisição dos medicamentos e em ambos os grupos existe altos índices de desinformação sobre a doença e termos técnicos independente do tempo de tratamento.Aproximadamente, $50 \%$ dos entrevistados descontinuaram o tratamento no primeiro ano de diagnostico e são os responsáveis pela administração dos seus medicamentos. Afirmam dificuldade ao acesso as consultas com 30\% dizendo ser muito difícil e outros $25 \%$, ser

Palavras-chave: Hipertensão; Programa

Saúde da Família.
Key Words: Hypertension; Family Health

Program.

${ }^{1}$ Acadêmicos da Faculdade de Medicina de Barbacena

${ }^{2}$ Preceptora das disciplinas de Clínica Médica e Cardiologia da Faculdade de Medicina de Barbacena; Mestre em Medicina pela Universidade Federal de Minas Gerais (UFMG). Especialista em Cardiologia pela Sociedade Brasileira de Cardiologia e Associação Médica Brasileira; Especialista em Clínica Médica pela Sociedade Brasileira de Clínica Médica e Associação Médica Brasileira. 
difícil. Dos 69,3\% dos integrantes do grupo não aderente contra $51,2 \%$ do grupo aderente deixaram de tomar os seus medicamentos alguma vez $\left(X^{2}=9,34 ; \mathrm{p}=0,002\right)$, sendo essa questão que separou os que aderem e não aderem. Esses responderam ao item com as seguintes respostas: "por vezes, raramente e nunca" e aqueles com: "sempre, quase sempre e com freqüência". Sobre a dieta, 49,5\% dos que não aderem informam não seguir as orientações, contrapondo com 35,5\% dos pacientes incluídos no grupo que adere $\left(X^{2}=5,69 ; p=0,017\right) . A$ adesão ao tratamento nas unidades de PSF foi maior quando comparado ao centro especializado (NAHI) e em ambos os grupos existe uma preocupação quanto aos riscos da doença já que 75\% dos entrevistados retornam as consultas marcadas. Já quanto à assiduidade as reuniões sobre HAS na unidade de tratamento, $74,3 \%$ dos pacientes que não seguem o tratamento informam que não freqüentam as palestras enquanto 40,8\% dos aderentes comparecem as reuniões agendadas pela unidade de tratamento que pertence. Nesse grupo, existe uma maior preocupação na realização de exames de rotina $\left(\mathrm{X}^{2}=\right.$ $22,35 ; \mathrm{p}=0,000)$ e aproximadamente $80 \%$, conhecem a importância da adesão ao tratamento e o risco da não adesão contra $64,4 \%$ dos inclusos no grupo não aderente $\left(\mathrm{X}^{2}=\right.$ $10,72 ; \mathrm{p}=0,001)$.

CONCLUSÃO: As unidades de PSF e do NAHI possuem alta adesão ao tratamento (cerca de $75 \%$ ) mas a aderência é maior no PSF comparado ao NAHI. Provavelmente tais índices sejam justificados pelo acompanhamento mais próximo das famílias residentes na região.

\section{Introdução}

A hipertensão arterial sistêmica (HAS) é uma situação clínica de natureza multifatorial, caracterizada por níveis elevados de pressão arterial (maiores que 140 / 90 mmhg) ${ }^{1}$. Ela acomete aproximadamente $25 \%$ da população adulta. É um dos principais fatores de risco para doenças cárdio e cerebrovasculares, sendo o principal fator de risco para o acidente vascular cerebral ${ }^{2}$.

O tratamento da HAS se baseia no uso de medicamentos anti-hipertensivos e em medidas não-farma- cológicas. O tratamento não-farmacológico, com base em modificações do estilo de vida, está indicado para todos os hipertensos e para normotensos com fatores de risco cardiovascular ${ }^{3}$.

Define-se adesão aos tratamentos o grau de concordância entre as recomendações do prestador de cuidados de saúde e o comportamento do paciente em relação ao regime terapêutico proposto ${ }^{4}$. Identifica-se que, da população hipertensa, apenas 50\% têm o diagnóstico; destes, $50 \%$ são tratados, e, dos tratados, $50 \%$ têm sua pressão adequadamente controlada. Estes índices sugerem que apenas um em cada oito pacientes é efetivamente tratado ${ }^{5}$.

Várias barreiras podem ser identificadas na adesão ao tratamento anti-hipertensivo. Em relação à doença: cronicidade, ausência de sintomatologia específica e a possibilidade de complicações tardias ${ }^{6}$. Quanto ao paciente: idade, sexo, raça, escolaridade, ocupação, estado civil, religião, hábitos de vida, aspectos culturais, crenças e contexto socioeconômico. Muitas vezes, a desinformação acarreta no paciente o medo de intoxicação, hipotensão, interação medicamentosa, além do conceito equivocado de que, após a normalização dos níveis pressóricos, a necessidade do tratamento inexiste ${ }^{7}$.

Em 1994, foi criado pelo Ministério da Saúde o Programa Saúde da Família (PSF), tendo como objetivo principal a reorganização da prática da saúde, visando à melhoria da qualidade de vida dos brasileiros por meio do conhecimento da realidade das famílias e da avaliação de suas características sociais ${ }^{8}$.

Em Barbacena, desde novembro de 2002, os hipertensos contam com atendimento e acompanhamento especializados no Núcleo de Atendimento ao Hipertenso (NAHI), que oferece consultas médicas especializadas, destacando-se como referência no diagnóstico e tratamento da hipertensão arterial neste município.

O objetivo deste trabalho é determinar o conjunto de fatores que interferem na adesão ao tratamento de pacientes com hipertensão arterial sistêmica atendidos em um serviço especializado (Núcleo de Atendimento ao Hipertenso) e no Programa Saúde da Família, no município 
de Barbacena.

\section{Métodos}

Trata-se de um estudo de corte transversal, realizado por meio de inquérito (questionário contendo 46 questões) com pacientes hipertensos, selecionados entre os 13.000 pacientes inscritos no NAHI e PSF do município de Barbacena, no período de fevereiro a agosto de 2005.

A amostra foi constituída por 400 pacientes, com idade superior a 18 anos, selecionada a partir de uma listagem dos pacientes atendidos pelo NAHI e PSF, sorteandose o primeiro paciente e sendo os demais escolhidos sequencialmente a cada dez nessa mesma listagem. Quando não era possível localizar ou identificar o paciente escolhido pelo critério citado, utilizava-se o primeiro nome que vinha imediatamente depois na lista. A pressão arterial foi aferida por três vezes durante a entrevista (início, meio e final), utilizando-se manômetros aneroides e estetoscópios, segundo a classificação das IV Diretrizes Brasileiras de Hipertensão Arterial da Sociedade

A classificação dos pacientes quanto à adesão ou não ao tratamento da HAS foi realizada pela análise do item 27 do questionário: "Você toma os medicamentos todos os dias na hora certa?". Quando as respostas eram "sempre", "quase sempre" e "com frequência", os pacientes eram incluídos no grupo adesão, e, quando as respostas eram "por vezes", "raramente" e "nunca", eram considerados não-aderentes ao tratamento. $\mathrm{O}$ nível de significância estatístico adotado na análise foi de $5 \%$.

\section{Resultados e Discussão}

Foram coletados 400 questionários, sendo 154 $(38,5 \%)$ no NAHI e $246(61,5 \%)$ no PSF. Os questionários foram divididos em dois grupos de acordo com a resposta dada à questão número 27 , sendo que 101 pacientes $(25,2 \%)$ foram incluídos na categoria não-aderente e 299 (74,7\%), na aderente ao tratamento. Cerca de 16 a 50\% descontinuam a medicação anti-hipertensiva durante o primeiro ano de uso, e um número substancial daqueles que permanecem em uso da medicação o fazem de modo inadequado ${ }^{9}$ - concordante com o presente estudo.

A totalidade da amostra foi constituída por indivíduos hipertensos, com idade média de 58,7 anos e desvio padrão de 11,7 , entre os que aderem ao tratamento, e idade média de 58,3 anos entre o grupo aderente ao tratamento e desvio padrão de 12,7, $\mathrm{p}=0.7931$. Em ambos os grupos prevalecem indivíduos com mais de 50 anos de idade, o que indica que a HAS é uma doença que acomete mais adultos e idosos.

Há neste estudo grande predomínio de mulheres, indivíduos da raça branca, casados e católicos. Esses resultados mostram que não há relação estatística entre o fato de aderir ou não ao tratamento em qualquer uma das quatro variáveis, em concordância com a literatura ${ }^{10,11}$

A maioria dos hipertensos não faz exercício físico, não ingere bebida alcoólica e não fuma - e não há relação estatística entre o fato de aderir ou não ao tratamento com qualquer uma das variáveis citadas. Considerando que o tratamento anti-hipertensivo depende, além dos medicamentos, da mudança do estilo de vida, é preocupante constatar que os hipertensos de Barbacena não fazem exercício físico. Em estudo de metanálise, o exercício físico aeróbico resultou em redução da pressão arterial de $3 / 4 \mathrm{mmHg}$ em normotensos, 6/7 mmHg em hipertensos limítrofes e 10/ $8 \mathrm{mmHg}$ em hipertensos. ${ }^{3,12}$ Por outro lado, é confortante que, em ambos os grupos, os índices de não-etilistas e nãotabagistas sejam elevados. Observando-se 14.125 homens com história pregressa de HAS e sem outras comorbidades por 5,4 anos, constatou-se que a abstenção da ingestão de bebida alcoólica reduz a mortalidade cardiovascular RR $0,56(95 \% \text { IC }=0,49 \text { a } 0,77)^{1,13}$.

A maioria dos pacientes afirma ter apresentado sintomatologia pré-tratamento (palpitação, cefaleia, vertigem e outros) - provavelmente decorrente dos níveis tensionais elevados, já que estes são os sintomas mais comuns. Após a instituição da terapêutica e/ou descobrimento da causa dos sintomas, os pacientes em ambos os grupos afirmaram que houve uma redução dos sintomas, mostrando que mesmo o grupo não-aderente deve se submeter a alguma forma de tratamento, mesmo que irregular. 
Segundo estudo, ${ }^{10}$ os efeitos colaterais dos medicamentos anti-hipertensivos tais como edema de membros inferiores, tosse e disfunção erétil, entre outros, contribuem para a diminuição da adesão. Em um estudo, ${ }^{9}$ eles representaram a segunda causa de abandono. Em outra citação ${ }^{10}$, $37 \%$ dos pacientes controlados abandonaram a terapêutica devido aos efeitos colaterais.

No presente estudo, não foi medido o papel dos efeitos colaterais na adesão ou não-adesão ao tratamento. Entretanto a não-existência de diferenças entre aderentes e não-aderentes observada aqui quanto aos sintomas reportados durante o tratamento indicam que possíveis efeitos colaterais da medicação não tiveram importância na determinação de quem adere ou não adere ao tratamento em Barbacena, pois alguns dos sintomas levantados neste estudo estão associados à ocorrência dos efeitos colaterais da medicação.

A maioria dos pacientes de ambos os grupos adquirem os seus medicamentos anti-hipertensivos na unidade de tratamento (40,5\% no grupo aderente e 46,5\% no grupo não-aderente). A grande maioria dos pacientes (71,0\%) respondeu ter dificuldade em comprar seus medicamentos, o que condiz com a realidade econômica do país, já que, 79,2\% da amostra recebem menos de dois salários mínimos.

Entre os dois grupos não há predomínio do uso de outra terapia durante o tratamento anti-hipertensivo. $\mathrm{O}$ uso de outra terapêutica poderia dificultar a aderência do paciente ao tratamento anti-hipertensivo, pois facilitaria o confundimento na hora da administração de seus medicamentos. Mas o presente estudo contraria este dado, já que não há relação estatística entre o fato de aderir ou não ao tratamento sobre este quesito.

Os pacientes foram questionados sobre o conhecimento do significado de intoxicação, hipotensão, interação medicamentosa e efeitos colaterais; houve grande predomínio do desconhecimento de tais termos técnicos. Esses dados permitem concluir que os pacientes de ambos os grupos algumas vezes não entendem as recomendações médicas e/ou não elucidam suas dúvidas com os profissionais de saúde. Em um estudo ${ }^{9}$, entre as razões mais citadas pelos pacientes para deixar de tomar o medicamento, está a falta de comunicação adequada entre o paciente e o seu médico em relação à doença e as sérias consequências do abandono do tratamento. Isso pode ocorrer devido à insuficiência de informação prestada ou à incapacidade de percepção do paciente.

A adesão ao tratamento independe do tempo de conhecimento da HAS entre os que aderem e não aderem $(p=0,511$ IC $=0,51$ a 1,36$)$. No estudo, ${ }^{10}$ é visto que menos tempo de doença associa-se ao abandono e ao controle inadequado da pressão arterial, o que contradiz o presente estudo.

A adesão ao tratamento independe do tempo de tratamento da doença $(p=0,695 \mathrm{IC}=0,53$ a 1,46). Em um estudo $^{15}$ identificaram que apenas $78 \%$ dos pacientes que iniciaram a terapêutica anti-hipertensiva continuaram a medicação após um ano de tratamento e que, decorridos quatros anos e meio, somente $46 \%$ ainda persistiam no tratamento. De acordo com os percentuais encontrados nesta pesquisa, é preocupante que mais de 50\% dos pacientes descontinuem o tratamento anti-hipertensivo já no início da doença, podendo ter complicações mais precoces.

Em ambos os grupos, 84\% dos entrevistados são os próprios responsáveis pela administração de seus medicamentos. Esse resultado sugere que a família desconheça a necessidade de sua ajuda e da importância deste fato na adesão ao tratamento anti-hipertensivo.

Sobre a interrupção do tratamento devido a reações adversas e/ou falta de medicamento na unidade de tratamento e/ou sem orientação médica em ambos os grupos, mostrou-se que a maioria não interrompe o tratamento pelas causas citadas $(\mathrm{p}=0,624 ; \mathrm{p}=0,760 ; \mathrm{p}=0,960$ respectivamente). Resultado confortante, já que, mesmo entre o grupo não-aderente ao tratamento, parece existir uma preocupação com a doença e suas complicações.

Em ambos os grupos, os percentuais foram altos para a afirmação de que um maior conhecimento sobre a doença facilita a adesão ao tratamento (acima de 90,0\%), ou seja, mesmo com percentual alto $(80,0 \%)$ de pacientes que afirmam deter um bom conhecimento sobre a impor- 
tância do tratamento, a população hipertensa de Barbacena se sente carente de informações mais didáticas a respeito da doença.

Quando analisada a presença de caminhada, ginástica e reuniões para orientação dietética na unidade de tratamento, houve grande predomínio da não-promoção dessas atividades em ambos os grupos. No estudo DEW - IT (Results of the Diet, Exercise, and Weigt Loss Intervention Trial) ${ }^{3,16}$, foram avaliados os efeitos da mudança de estilo de vida na pressão arterial e nos fatores de risco cardiovascular em 44 pacientes. Os pacientes foram subdivididos para um grupo de intervenção e para um grupo controle. A pressão arterial de vigília pela monitorização ambulatorial da pressão arterial (MAPA) foi reduzida para 12,1/6,6 mmHg. Apesar de os resultados do trabalho acima citado ilustrarem o potencial da mudança do estilo de vida como terapia eficaz, o presente estudo mostrou que a promoção da mudança do estilo de vida nos dois serviços não alcançou bons resultados, já que os grupos aderente e não-aderente não se mostraram diferentes entre os quesitos citados, indicando que só a promoção não é suficiente, mas que é necessário promover maior conhecimento da necessidade da mudança do estilo de vida e busca ativa dos pacientes para que esses participem.

O fato de ter ou não especialista na unidade de tratamento não interferiu na adesão dos pacientes pesquisados. Tal resultado no presente estudo é preocupante, pois mostrou que os especialistas das unidades estudadas em Barbacena não estão atingindo os seus objetivos na ajuda aos pacientes na continuidade correta de seu tratamento, embora sejam os profissionais mais indicados para recomendar o tratamento.

Quanto ao acesso às consultas, foi encontrado um predomínio das respostas "muito difícil" (mais de 30\%) e "difícil" (mais de 25\%) em ambos os grupos. Mas tal dificuldade não influenciou na adesão ao tratamento $-\mathrm{p}=$ 0,709 .

Quanto à aferição das pressões arteriais sistólica e diastólica, existiu predomínio da hipertensão arterial sistólica leve e pressão diastólica normal, de acordo com a IV Dire- trizes Brasileiras de Hipertensão Arterial da Sociedade Brasileira de Cardiologia ${ }^{9} \mathrm{em}$ ambos os grupos. Apesar de 1/ 4 dos pacientes deste estudo ser considerado não-aderente, seus níveis pressóricos se encontram levemente alterados. Certamente, se optassem por seguir corretamente as recomendações propostas pelos médicos para o tratamento anti-hipertensivo, estes teriam suas pressões tanto sistólica quanto diastólica normalizadas, possuindo assim um menor risco de ocorrência de picos hipertensivos e, desta forma, retardariam as complicações cardio e cerebrovasculares advindas da doença.

A figura 1 apresenta as frequências das respostas dadas pelos pacientes entrevistados ao quesito "se deixa ou não de tomar os seus medicamentos". A aferição da significância estatística realizada na comparação dos percentuais exibidos no gráfico apresentou $\mathrm{X}^{2}=9,34$ e $\mathrm{p}=0,002$. As razões de probabilidades observadas para esses percentuais foram de 0,28 a 0,77 .

Figura 1. Distribuição das frequências das respostas dadas pelos pacientes entrevistados à questão: "Já deixou de tomar os seus medicamentos alguma vez?”.

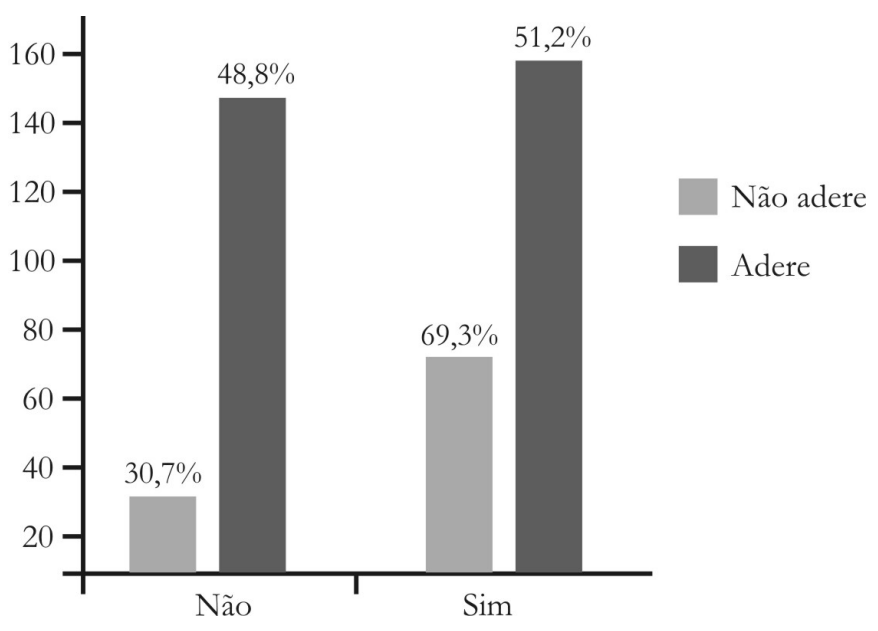

O percentual dos que afirmam "já terem deixado de tomar os seus medicamentos" entre os não-aderentes foi maior do que a resposta "não deixam de tomar os seus medicamentos". A diferença entre os dois grupos nessa comparação foi estatisticamente significativa. Isso significa 
que os que aderem não deixam de tomar os medicamentos em uma porcentagem maior comparado ao outro grupo.

De acordo com o que está relatado no presente trabalho esta pergunta serviu para dividir os grupos em aderente e não-aderente, segundo as respostas dadas pelos entrevistados. Em um estudo ${ }^{11}, 69 \%$ do grupo não-controlado deixam de tomar o medicamento, enquanto $31 \%$ do grupo controlado diz deixar de tomar o medicamento.

A frequência das respostas encontradas nesse quesito é alarmante, pois mesmo o grupo aderente $(51,2 \%)$ afirma já ter deixado de usar a terapêutica indicada. Com isso, a promoção ao tratamento, em ambos os grupos, encontra-se deficiente, pois os pacientes ainda resistem em fazer o correto seguimento das prescrições médicas. Tal conduta talvez possa ser explicada pela falta de interesse ou desinformação sobre os verdadeiros perigos da não-adesão ao tratamento.

A figura 2 mostra as frequências das respostas dadas pelos entrevistados sobre a realização de dieta. A aferição da significância estatística realizada na comparação dos percentuais exibidos no gráfico apresentou $\mathrm{X}^{2}=5,69$ e $\mathrm{p}=$ 0,017. As razões de probabilidades observadas para esses percentuais foram de 0,34 a 0,91 .

Figura 2. Distribuição das frequências das respostas dadas pelos pacientes entrevistados à questão: "Faz a dieta recomendada?".

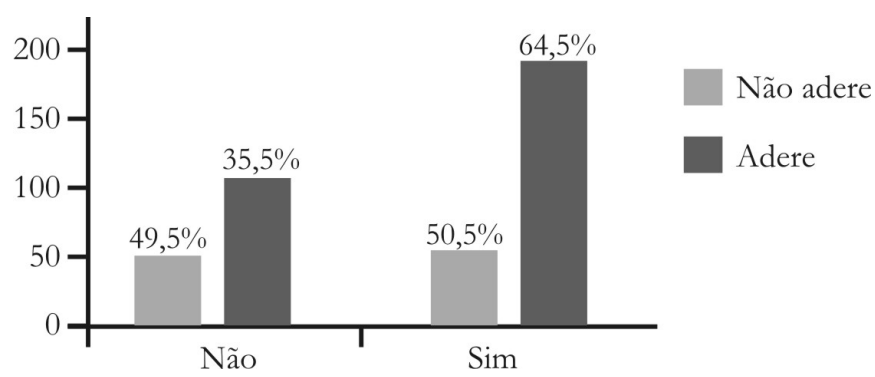

A diferença entre os dois grupos nesta comparação foi estatisticamente significativa. Os resultados deste estudo mostram que mais de $50 \%$ dos participantes de ambos os grupos praticam a dieta, mas o grupo aderente apresenta índices maiores.

O bom controle da hipertensão arterial tem uma forte correlação com a adesão a um plano alimentar adequa$\mathrm{do}^{16,17}$, o que foi confirmado neste estudo, já que os aderentes possuem um maior percentual relacionado ao quesito fazer dieta. Essa diferença entre os grupos aderente e nãoaderente mostra que os aderentes preocupam-se mais em realizar uma adequada dieta e que provavelmente conhecem sobre a importância desta associação, mesmo que a façam de forma irregular. Com a implementação de mais informações sobre este assunto aos grupos talvez seja possível evitar e/ou retardar os malefícios da hipertensão em longo prazo.

A figura 3 apresenta as frequências das respostas dadas pelos hipertensos entrevistados sobre o local de tratamento. A aferição da significância estatística realizada na comparação dos percentuais exibidos no gráfico apresentou $X^{2}=6,30$ e $p=0,012$. As razões de probabilidades observadas para esses percentuais foram de 1,85 (1,2 $-3,0)$.

Figura 3. Distribuição das frequências das respostas dadas pelos pacientes entrevistados à questão: "Onde faz seu controle da HAS?".

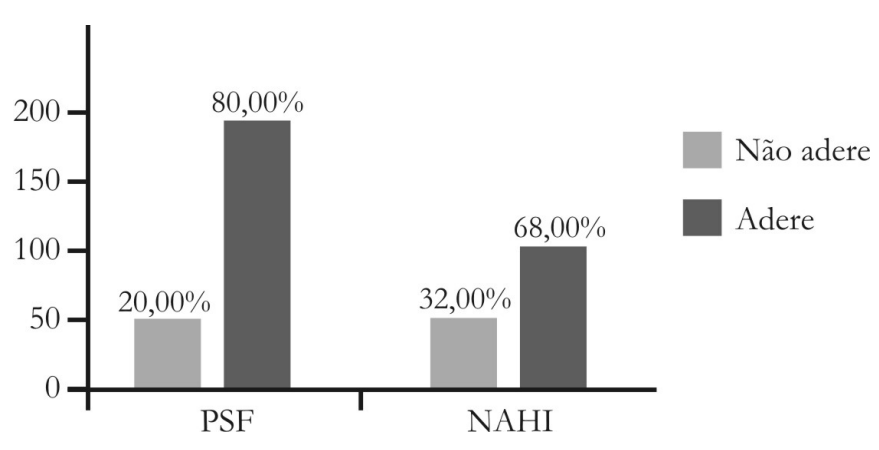

A comparação dos dois grupos quanto à unidade de tratamento mostra altos percentuais de adesão no NAHI e PSF.

Apesar de o NAHI ser um centro especializado no tratamento de HAS, seu percentual de pacientes aderentes não se sobressaiu ao número de aderentes do PSF; resultado 
que mostra que, apesar de o NAHI possuir diversas especialidades para o melhor tratamento da HAS, este não está preparado para ajudar o paciente a ter uma maior adesão ao tratamento, enquanto o PSF, apesar de suas dificuldades em ter especialistas presentes na unidade, mostra-se mais eficaz em ajudar o paciente a manter um correto tratamento. Talvez tal fato se deva às visitas constantes de agentes de saúde na residência dos pacientes para avaliar seu estado geral, o que é transmitido à enfermagem e aos médicos que decidem sua conduta. Não foram encontrados dados na literatura pesquisada sobre esse quesito.

A figura 4 apresenta as frequências das respostas dadas pelos hipertensos entrevistados sobre o retorno às consultas. A aferição da significância estatística realizada na comparação dos percentuais exibidos no gráfico apresentou $\mathrm{X}^{2}=5,31 \mathrm{e} \mathrm{p}=0,021$. As razões de probabilidades observadas para esses percentuais foram de 0,27 a 0,91 .

Figura 4. Distribuição das frequências das respostas dadas à questão: "Você retorna às consultas?".

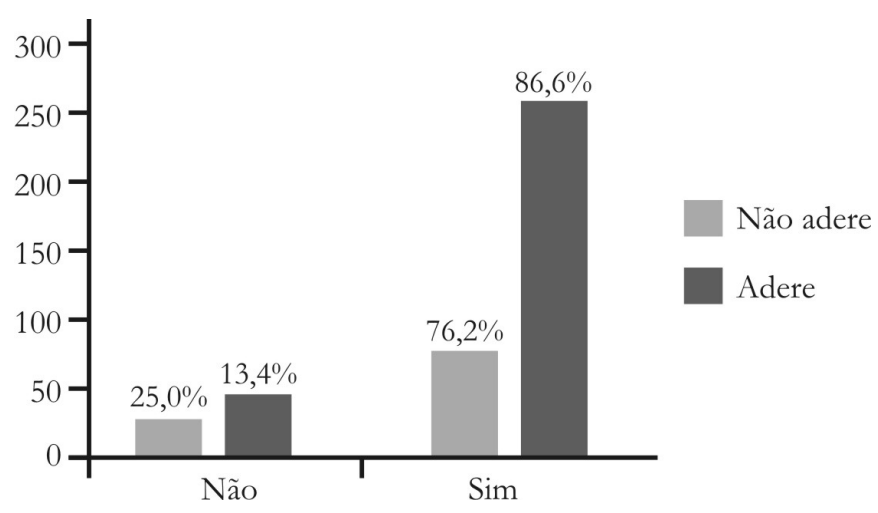

A diferença entre os dois grupos nesta comparação foi estatisticamente significativa. Em ambos os grupos, mais de $75 \%$ dos pacientes retornam às consultas.

A assiduidade às consultas demonstra que os pacientes têm a percepção de serem portadores de uma condição de saúde que inspira cuidados médicos; esse comportamento está associado a uma melhor taxa de controle da pressão arterial e de uma melhor aderência ao tratamento ${ }^{18}$, fato confirmado no atual estudo que mostrou uma maior assiduidade às consultas entre o grupo aderente ao tratamento. O presente estudo mostra também uma alta taxa de regularidade nas visitas ao médico no grupo não-aderente, o que mostra que os pacientes em ambos os grupos se preocupam com sua doença, mesmo não seguindo, ou não conseguindo cumprir, recomendações propostas. Nesse cenário, as informações prestadas aos hipertensos podem ser passadas de forma técnica e generalizada, dificultando a adesão, pois o ideal seria que as recomendações fossem específicas para cada paciente de acordo com sua realidade.

A figura 5 apresenta as frequências das respostas dadas pelos hipertensos entrevistados sobre a assiduidade às reuniões sobre HAS na unidade de tratamento. A aferição da significância estatística realizada na comparação dos percentuais exibidos no gráfico apresentou $\mathrm{X}^{2}=6,71$ e $\mathrm{p}=$ 0,009 . As razões de probabilidades observadas para esses percentuais foram de 0,17 a 3,39 .

Figura 5. Distribuição das frequências das respostas dadas pelos pacientes à questão: "Frequenta as reuniões sobre HAS na unidade de tratamento?”.

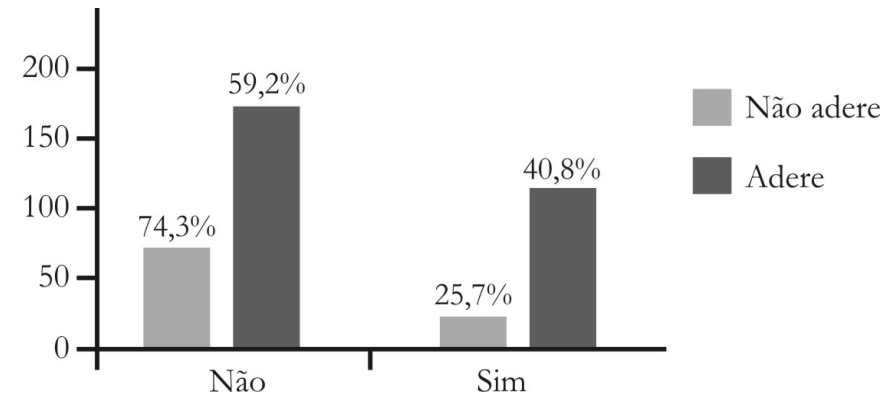

De acordo com os percentuais vistos neste quesito, observa-se que os aderentes têm tendência a se reunir mais frequentemente para discutir sobre a sua doença dos que os não-aderentes. Entretanto, a maioria em ambos os grupos não frequentam as reuniões promovidas pela unidade de tratamento sobre HAS. Esses números apontam para a dificuldade que os pacientes possuem em disponibilizar tempo para escutar as palestras de especialistas e outros profis- 
sionais de saúde sobre a melhor forma de controlar os níveis pressóricos. Outro aspecto que pode ter influenciado nesses é a falta de informação sobre dia, hora e local das reuniões. Mesmo que haja preocupação por parte do paciente em relação à sua saúde, a deficiência do serviço prestado pela unidade de tratamento em comunicá-los prejudica-os em aderir ao tratamento. Não foram encontrados da- dos na literatura pesquisada sobre esta variável.

A figura 6 apresenta as frequências das respostas dadas pelos entrevistados sobre a dificuldade na realização dos exames de rotina em sua unidade de tratamento. A aferição da significância estatística realizada na comparação dos percentuais exibidos no gráfico apresentou $\mathrm{X}^{2}=22,35$ e $\mathrm{p}=0,000$. As razões de probabilidades observadas para esses percentuais foram de 1,97 a 5,76.

Figura 6. Distribuição das frequências das respostas dadas pelos entrevistados à questão: "Você faz exames de rotina?".

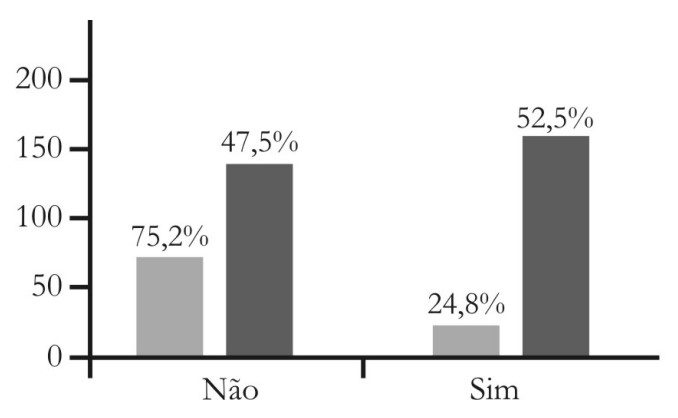

A diferença entre os dois grupos nesta comparação foi estatisticamente significativa. Isto significa que os aderentes realizam mais exames de rotina que os não-aderentes, o que revela uma maior preocupação na prevenção de complicações devido à sua doença de base.

A grande maioria dos não-aderentes mostra-se despreocupada com o controle das complicações da HAS - a soma da pouca assiduidade às consultas com a baixa procura de realização de exames mostra que este grupo possui um maior risco de desenvolver mais precocemente os malefícios dos altos níveis pressóricos. A importância na realização de exames regularmente se faz por conta do diagnóstico precoce de outras comorbidades geradas pela própria HAS ou que juntas possam levar a um prejuízo maior à saúde do paciente hipertenso. O diagnóstico precoce, muitas vezes, evita despesas desnecessárias e até mesmo complicações mais severas de difícil resolução.

A figura 7 mostra a frequência das respostas dadas pelos entrevistados sobre o conhecimento da importância do tratamento anti-hipertensivo e os riscos da não-adesão ao tratamento. A aferição da significância estatística realizada na comparação dos percentuais exibidos no gráfico apresentou $\mathrm{X}^{2}=10,72$ e $\mathrm{p}=0,001$. As razões de probabilidades observadas para esses percentuais foram de 0,39 a 0,76 .

Figura 7. Distribuição das frequências das respostas dadas pelos pacientes entrevistados à questão: "Você conhece a importância do tratamento anti-hipertensivo e os riscos da não-adesão ao tratamento?”.

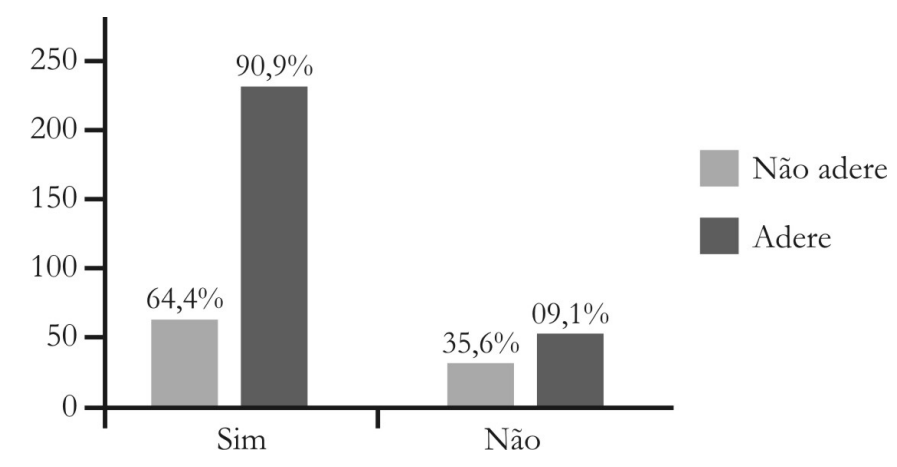

A diferença entre os dois grupos nesta comparação foi estatisticamente significativa. Isto significa que o grupo aderente procura obter maior conhecimento sobre a sua doença de base e consequentemente da importância de seu tratamento e adesão.

Apesar de mais de 50\% do grupo nãoaderente afirmar conhecer a importância e os riscos da nãoadesão ao tratamento, estes parecem apresentar dificuldades em seguir essas informações, ficando assim mais susceptíveis às complicações da HAS. O conhecimento sobre a importância do tratamento é importante, pois os pacientes conhecem os reais malefícios da não-adesão ao tratamento 
e com isso procuram realizar uma terapia anti-hipertensiva mais eficaz e continuada. Não foram encontrados dados na literatura pesquisada.

\section{Conclusão}

O estudo apresenta alta taxa de adesão ao tratamento da hipertensão arterial sistêmica entre os pacientes hipertensos de Barbacena, cerca de 75\% da amostra.

Comparando a adesão entre os pacientes do PSF e do NAHI, a taxa de aderência é cerca de $12 \%$ mais alta no PSF. Talvez tal fato se deva à busca ativa aos domicílios dos pacientes, através dos agentes de saúde, realizado pelo PSF.

É notável no estudo e levantamento bibliográfico a importância de orientar e educar os pacientes sobre sua patologia, possíveis complicações, tratamento e importância a adesão deste. Ainda é necessário individualizar a orientação do paciente conforme seu vocabulário, condições sócioeconômicas e atividades diárias.

De forma semelhante se faz necessário integrar o paciente à unidade de saúde, atividades propostas pela unidade e comunidade, a fim de evitar burocracias no atendimento, marcação de consultas e acesso a informação.

\section{Referências}

\section{IV DIRETRIZES BRASILEIRAS DE HIPERTENSÃO} ARTERIAL, 2002, Campos do Jordão. Anais... Campos do Jordão: SBH, 2002. Disponível em: <http:// www.sbh.org.br/documentos/index.asp > . Acesso em 03 set. 2004

2.AMODEO. Celso. Hipertensão arterial: prognóstico e epidemiologia. In: TIMERMAN, Ari; CÉSAR, Luiz Antônio Machado (Ed.). Manual de cardiologia SOCESP. São Paulo: Atheneu, 2000. Cap71, p. 307-309.

3..LOPES, Heno Ferreira; BARRETO-FILHO, José Augusto S.; RICCIO, Grazia Maria Guerra. Tratamento não-medicamentoso da hipertensão arterial. Revista da Sociedade de Cardiologia do Estado de São Paulo, São Paulo, v.13, n.1, p.148-155, jan. / fev. 2003.
4.DELGADO, Artur Barata; LIMA, Maria Luisa. Contributo para a validação concorrente de ma medida de adesão aos tratamentos. Psicologia, Saúde e Doenças, Lisboa, v.2, n.2, p.81-100, 2001. 5.GIORGI, Dante. O problema da adesão ao tratamento. [nov.2003]. Entrevistador: Sociedade Brasileira de Hipertensão. [s.1.], 2003. Disponível em: < http:/ /www.sbh.org.br/entrevistas/entrevista7.htm>. Acesso em: 03 set. 2004.

6.SARQUIS, Leila Maria Mansano; DELLÁCQUA, Magda Cristina Queiroz; GALLANI, Maria Cecília Bueno Jayme; MOREIRA, Rosa Maria; BOCCHI, Silvia Cristina Mangini, TASE, Terezinha Hideco; PIERIN, Ângela Maria Geraldo. A adesão ao tratamento na Hipertensão Arterial: Análise de produção científica. Esc. Enfermagem USP, São Paulo, V.32, n.4, p335-53, dez.1998.

7.BUSNELLO, Renné Gusmão. et al. Características associadas ao abandono do acompanhamento de pacientes hipertensos atendidos em um ambulatório de referência. Arquivos Brasileiros de Cardiologia. Campinas, v.76, n.5, p.349351,2001

8.BRASIL. Ministério da Saúde. Programa saúde da família. Brasília: Ministério da Saúde, [s.d.]. Disponível em:<http:/ /portal.saude.gov.br/saude/visao.cfm?id_area $=149>$. Acesso em: 21 ago.2004.

9.ANDRADE, Jadelson P., et al. Aspectos epidemiológicos da aderência ao tratamento da hipertensão arterial sistêmica. Arquivos Brasileiros de Cardiologia. Campinas, v.79, n.4, p.375379, 2002.

10.STRELEC, Maria Aparecida A. Moura; PIERIN, Ângela M. G.; MION JR, Dicio. A influência do conhecimento sobre a doença e a atitude Frente à Tomada dos Remédios no Controle da Hipertensão Arterial. Arquivos Brasileiros de Cardiologia, São Paulo, V.81, n.4, p 343-8, 2003. 11.FUCHS, Sandra Costa; PETTER Juliano G., ACCORDI, Melissa C.; ZEN, Vanessa L.; PIZZOL-JR, Antônio D.; Moreira, Leila Beltrami; FUCHS, Flávio Danni. Estabelecendo a Prevalência de Hipertensão Arterial Sistêmica. Influência dos Critérios de Amostragem. Arquivos Brasileiro Cardiologia, Porto Alegre, V.76, n.6, p.445-8, 2001 12.SOUZA, Weimar Sebba Barroso de; AMARAL, 
Geraldo Francisco do. Álcool, hipertensão arterial e doença cardiovascular. Revista Brasileira de Hipertensão, Ribeirão Preto, vol. 11(2):112-11,2004.

13.CONCEIÇÂO, Tatiana Valverde da; GOMES, Fabiano Alves; TAUIL, Pedro Luiz; ROSA, Tânia Torres. Valores e Pressão Arterial e suas Associações com Fatores de Risco Cardiovasculares em Servidores da Universidade de Brasilia. Arquivos Brasileiros de Cardiologia, Brasília, V.86, n.1, p26-31, jan.2006.

14.ROCHA, Augusto. Adesão ao tratamento: o papel do médico. Revista Brasileira de Hipertensão, Ribeirão Preto, v.10, n.3, p.213-215, jul. /set. 2003. Disponível em: <http:// departamentos.cardiol.br/DHA/publicações/10-3/ adesao.pdf >. Acesso em: 03 set. 2004.

15.JARDIM, Aline Rodrigues; MOURA, Luciana Fonseca de; SILQUEIRA, Salete Maria de Fátima. A Importância de uma assistência diferenciada ao cliente hipertenso visando à adesão ao tratamento proposto. Anais do $8^{\circ}$ Encontro de Extensão da UFMG, Belo Horizonte, 03 a 08 de outubro de 2005.

16.MONEGO, Estelamaris Tronco; MAGGI, Cinthya. Gastronomia na promoção de saúde dos pacientes hipertensos. Revista Brasileira de Hipertensão, Ribeirão Preto, vol. 11(2): 105-108, 2004.

17.NONINO-BORGES, Carla Barbosa; DOS-SANTOS, José Ernesto. Terapia nutricional nas alterações metabólicas associadas à hipetensão arterial (diabetes melito, dislipidemias, hiperuricemia). Revista Brasileira de Hipertensão, Ribeirão Preto, vol 11(2) : 98-101, 2004.

18.COELHO, Eduardo Barbosa; NETO, Miguel Moysés; PALHARES, Raquel; CARDOSO, Maria Camila de Miranda; GELEILETE, Tufik José Magalhães, NOBRE, Fernando. Relação entre a Assiduidade às Consultas Ambulatoriais e o Controle da Pressão Arterial em Pacientes Hipertensos. Arquivos Brasileiros de Cardiologia, Ribeirão Preto, V.85, n3, p.157-161, set. 2005.

\section{Endereço para correspondência:}

Rua: Geraldino Lopes Canuto, 97

Barbacena- MG

CEP:36200-130

\section{Endereço eletrônico:}

julio_amaral456@hotmail.com 